Compliments of the Author.

$$
\text { tart I }
$$

THE ATTENUATION OF SOUND AND THE CONSTANT OF RADIATION OF AIR.

By A. WILMER DuFf.

(Reprinted from the Physical Revicw, Vol. VI, No. XXXII, March, I8g8.)

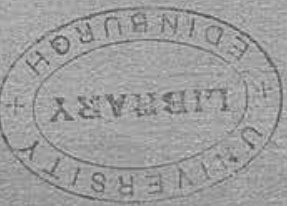




\title{
THE PHYSICAL REVIEW
}

\author{
A FOURNAL OF EXPERIMENTAL AND THEORETICAL PHYSICS \\ CONDUCTED BY \\ EDWARD L. NICHOLS \\ ERIEST MERRITT, AND FREDERICK BEDELL
}

Beginning with Volume V. (July-December, I897), two volumes of The Physical Review will be published annually, these volumes beginning in July and January, respectively, and containing at least five numbers each. The price of subscription is two dollars and fifty cents a volume (five dollars a year), or fifty cents a number. Subscriptions should be sent to the publishers, The Macmillan Companx, 66 Fifth Avenue, Nerw York; Messrs. Macmillan \& Co., LtD., London; or to Messts. Mayer and Mueller, Berlin.

Volumes I.-IV. of The Physical Review are annual volumes, each containing six bi-monthly numbers, beginning with the JulyAugust number, 1893 . These may be obtained from the publishers at the former subscription price, three dollars per volume.

Subscribers who desire to have their copies of the Review sent to them with the edges cut, can so obtain them by informing the publishers to that effect.

Correspondence relating to contributions should be addressed to the editors, at Ithaca, New York.

Manuscript intended for publication in The Physical Review must be communicated by the author; when publication in other journals is contemplated, notice to that effect should be given.

The authors of original articles published in the REviEW will receive one hundred separate copies in covers, for which no charge will be made; additional copies, when ordered in advance, may be obtained at cost.

\author{
PUBLISHED FOR CORNELL UNIVERSITY
}

THE MACMILLAN COMPANY, NEW YORK 
Deelaration

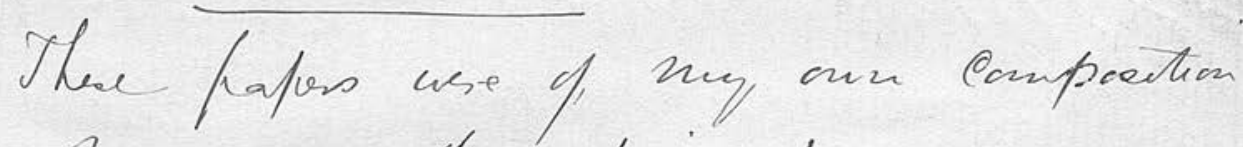
thrienfhout and the syperimental uote was

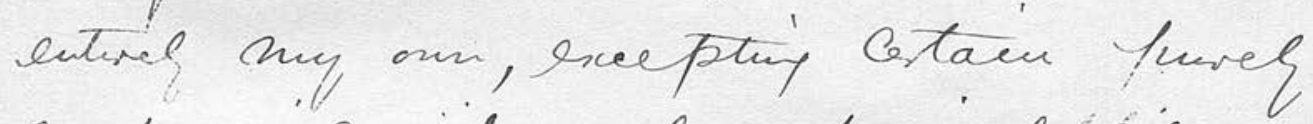
mederencal aid and eveh inderitiones to Other thapers as is ackuouleded, he rensiy theen fer the present purpose, I have correctid Gitaie nunor esrors and muspouts and adaies notes, pary explauatory and part ty referving to recent Cofrate futleations, and, in doing thes, I have ind hem indeltid te pugpestian from auyore.

In repard to the poncersal-meatefostery hinit of the nathematecal intioduction, I chould like te call attention its limited aine and preteuxion to originalit, as indisated under the beading "prel menary" in the fust paper.

Apid 19, 1901 


\title{
THE ATTENUATION OF SOUND AND THE CONSTANT OF RADIATION OF AIR.
}

\author{
By A. Wilmer Duff.
}

\section{Preliminary.}

T ${ }_{\text {se intensity }}^{1}$ of sound spreading in spherical waves from a in the passage, vary inversely as the square of the distance. It is certain, however, that a considerable proportion of the vibrational energy must in every second be converted into heat, though no attempt seems to have been made to determine experimentally how large this portion is. The chief purpose of the present paper is to describe some observations made by the author with a view to obtaining a rough estimate of this important quantity.

While this is probably the first attempt at an experimental investigation, several eminent physicists have discussed the question theoretically. Thus Stokes ${ }^{2}$ in I 845 studied the effect of the viscosity of the air and deduced numerical results, and in $185 \mathrm{I} \mathrm{he}^{3}$ found a formula for the effect of radiation from the condensations and to the rarefactions, but calculation from this formula is still impossible because of our ignorance of the rate of radiation of a gas. ${ }^{4}$

1 To avoid confusion I shall use " intensity" in the objective sense of flow of energy and loudness in the subjective sense.

2 Phil. Mag. (4), I., 305.

3 Cambridge Phil. Trans., VIII., 287.

${ }^{4}$ See remarks by Rayleigh in Nature, No. 1447, Vol. $5^{6}$ (1897).

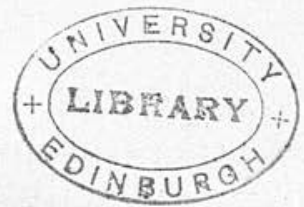


Rayleigh ${ }^{1}$ has applied Stokes' method to estimate the effect of conduction. All these investigations have referred to plane waves only. Kirchoff ${ }^{2}$ in 1868 discussed the effect of both conduction and viscosity on plane waves and indicated the result for spherical waves also. Brunhes ${ }^{3}$ has recently examined the effect of conduction on plane waves, obtaining a result in accord with those of Kirchoff and Rayleigh.

In order to deduce any intelligible results from the observations I have made, it is necessary to either assume or establish a law of diminution of intensity in spherical waves, taking account of viscosity, radiation, and conduction simultaneously. As considerable doubt might attach to a formula framed by combining the formulæ already obtained for the separate effects enumerated, it has seemed necessary to give the following brief theoretical discussion of the question.

\section{Theoretical.}

The dynamical equations of the small motions of a viscous compressible fluid are (in the alocue of ectraneons forces)

$$
\left.\begin{array}{l}
\frac{\partial u}{\partial t}+\frac{\mathrm{I}}{\rho_{0}} \frac{\partial p}{\partial x}=\frac{\mu}{\rho_{0}} \nabla^{2} u+\frac{\mu}{3 \rho_{0}} \frac{\partial}{\partial x}\left(\frac{\partial u}{\partial x}+\frac{\partial v}{\partial y}+\frac{\partial w}{\partial z}\right) \\
\frac{\partial v}{\partial t}+\frac{\mathrm{I}}{\rho_{0}} \frac{\partial p}{\partial y}=\frac{\mu}{\rho_{0}} \nabla^{2} u+\frac{\mu}{3 \rho_{0}} \frac{\partial}{\partial y}\left(\frac{\partial u}{\partial x}+\frac{\partial v}{\partial y}+\frac{\partial w}{\partial z}\right) \\
\frac{\partial w}{\partial t}+\frac{\mathrm{I}}{\rho_{0}} \frac{\partial p}{\partial z}=\frac{\mu}{\rho_{0}} \nabla^{2} w+\frac{\mu}{3 \rho_{0}} \frac{\partial}{\partial z}\left(\frac{\partial u}{\partial x}+\frac{\partial v}{\partial y}+\frac{\partial w}{\partial z}\right)
\end{array}\right\}
$$

where $\mu$ denotes the coefficient of viscosity and $\rho_{0}$ the normal density. Denoting a condensation by $s$, the equation of continuity is

$$
\frac{\partial u}{\partial x}+\frac{\partial v}{\partial y}+\frac{\partial w}{\partial z}+\frac{\partial s}{\partial t}=0
$$

This leads to an obvious simplification of (I). The above constitute four equations in five functions of $x, y, z$, and $t$, viz. : $u, v, w$, $p, s$. A fifth is supplied by the law of gaseous pressure

${ }^{1}$ Theory of Sound ( 1896$)$, Vol. II., p. 28.

2 Pogg. Ann., CXXXIV., 289.

${ }^{3}$ Jour. de Phys., VI., p. 289, 1897 (Abstract 473, Phys. Soc. of London, 1897). 


$$
p=\kappa \rho(\mathrm{I}+\alpha \theta)
$$

in which $\theta$ denotes excess of temperature. But, since $\rho$ denotes the density corresponding to a condensation $s$,

whence

$$
\begin{gathered}
\rho=\rho_{0}(\mathrm{I}+s), \\
p=k \rho_{0}(\mathrm{I}+s+\alpha \theta)
\end{gathered}
$$

the product of small quantities being neglected.

As this introduces a new function, $\theta$, another equation is needed. This is supplied by the thermal relation

$$
\frac{\partial \theta}{\partial t}=\beta \frac{\partial s}{\partial t}+\nu \nabla^{2} \theta-i \theta
$$

in which the first term on the right denotes the rate of change of temperature due to condensation, the second that due to conduction, and the third that due to radiation according to Newton's Law.

To adapt these equations to spherical waves, suppose the origin transferred to the source of sound; multiply (I) by $\frac{x}{r}, \frac{y}{r}, \frac{z}{r}$ respectively and add, and then transform by use of the relation

$$
r^{2}=x^{2}+y^{2}+z^{2} .
$$

Denoting the radial velocity by $V$ and writing $\mu^{\prime}$ for $\mu / \rho$, we get

$$
\frac{\partial V}{\partial t}+\frac{I}{\rho_{0}} \frac{\partial p}{\partial r}=\mu^{\prime} \frac{\partial}{\partial r}\left(\frac{\partial V}{\partial r}+2 \frac{V}{r}\right)-\frac{1}{3} \mu^{\prime} \frac{\partial^{2} s}{\partial r \partial t}
$$

From (2) and (4) by transformation and substitution of $\theta^{\prime}$ for $\theta / \beta$ we get

$$
\begin{gathered}
\frac{\partial V}{\partial r}+2 \frac{V}{r}+\frac{\partial s}{\partial t}=0 \\
\left(\frac{\partial}{\partial t}+\lambda\right) r \theta^{\prime}-\nu \frac{\partial^{2} \cdot r \theta^{\prime}}{\partial r^{2}}-r \frac{\partial s}{\partial t}=0
\end{gathered}
$$

By use of (3) and (6), (5) becomes

$$
\frac{\partial V}{\partial t}+\left(\kappa+\frac{4}{3} \mu^{\prime} \frac{\partial}{\partial t}\right) \frac{\partial s}{\partial r}+\kappa \alpha \beta \frac{\partial \theta^{\prime}}{\partial r}=0
$$


The motion here considered, being symmetrical about the origin, is necessarily irrotational and a velocity potential $\varphi$ exists which is a function of $r$ and $t$ only. On introducing it, (6), (7) and (8) become

$$
\begin{gathered}
\frac{\partial^{2} \cdot r \varphi}{\partial r^{2}}+\frac{\partial \cdot r s}{\partial t}=0 \\
\left(\frac{\partial}{\partial t}+\lambda\right) r \theta^{\prime}-\nu \frac{\partial^{2} \cdot r \theta^{\prime}}{\partial r^{2}}+\frac{\partial^{2} \cdot r \varphi}{\partial r^{2}}=0 \\
\frac{\partial \varphi}{\partial t}+\left(k+\frac{4}{3} \mu^{\prime} \frac{\partial}{\partial t}\right) s+\kappa \alpha \beta \theta^{\prime}=0
\end{gathered}
$$

An arbitrary function of $t$ has been omitted from (I I) as it may be supposed to be incorporated in $\varphi$ and to disappear on the restoration of $V$.

These equations being linear and the motion known to be periodic, we may assume that $\varphi, s, \theta^{\prime}$ contain $t$ only in virtue of the factor $\varepsilon^{h t}, h$ being a constant which will afterwards be regarded as imaginary. Hence

$$
\begin{gathered}
\frac{\partial^{2} \cdot r \varphi}{\partial r^{2}}+h r s=0 \\
(h+\lambda) r \theta^{\prime}-\nu \frac{\partial^{2} \cdot r \theta^{\prime}}{\partial r^{2}}+\frac{\partial^{2} \cdot r \varphi}{\partial r^{2}}=0 \\
h r \varphi+\left(k+\frac{4}{3} h \mu^{\prime}\right) r s+\kappa \alpha \beta r \cdot \theta^{\prime}=0
\end{gathered}
$$

Eliminating $r s$ and $r \theta^{\prime}$ and putting $\gamma=\mathrm{I}+\alpha \beta$ we get

$$
A \frac{\partial^{4} \cdot r \varphi}{\partial r^{4}}-B \frac{\partial^{2} \cdot r \varphi}{\partial r^{2}}+C \cdot r \varphi=0
$$

where

$$
\begin{gathered}
A=\frac{\nu}{h}\left(\kappa+\frac{4}{3} h \mu^{\prime}\right) \\
B=k \gamma+h\left(\frac{4}{3} \mu^{\prime}+\nu\right)+\frac{\lambda}{h}\left(k+\frac{4}{3} h \mu^{\prime}\right) \\
C=h^{2}\left(1+\frac{\lambda}{h}\right)
\end{gathered}
$$

Hence the required solution is $r \varphi=\varepsilon^{h t} \cdot \varepsilon^{q r}$, where $q$ satisfies the equation

$$
A q^{4}-B q^{2}+C=0
$$


Now for air at $0^{\circ} \mathrm{C}$. and atmospheric pressure the value of $\mu^{\prime}$ is: .132 and of $\nu .2567 .{ }^{1}$ Hence $\mu^{\prime}$ and $\nu$ may be regarded as small quantities. Our knowledge of the value of $\lambda$ is extremely vague. For notes of very low pitch $\lambda$ may be comparable in magnitude with the modulus of $h$; but for the very high-pitched notes employed in the second part of this paper (for which the modulus of $h=2 \pi \times$ 7000 ) it is evident that $\lambda$ cannot be comparable with the modulus of $h$; for if it were, a mass of air initially at any excess of temperature above the surrounding mass would fall, by radiation only, to onehalf of that excess in a length of time comparable with one sixtythousandth of a second. Hence we shall first solve (I6) for the case treated experimentally. This will enable us to make a rough estimate of the value of $\lambda$ and it will then be seen that the solution will hold for all sounds except those of the very lowest pitch.

Hence for the first approximation to $q$ we neglect $\mu^{\prime}, \nu$ and $\lambda / h$. Replacing $h$ by $n i$ we thus obtain

$$
q= \pm \frac{n i}{\sqrt{k \gamma}}
$$

To obtain the second approximation let

$$
q= \pm\left(\frac{n i}{\sqrt{k \gamma}}+m\right)
$$

and substitute, neglecting products of $\mu^{\prime}, \nu, \lambda / h$ and $m$. We thus. get

$$
m=\frac{2}{3} \frac{n^{2}}{a^{3}} \mu^{\prime}+\frac{\gamma-\mathrm{I}}{r} \frac{n^{2}}{2 a^{3}} \nu+\frac{\gamma-\mathrm{I}}{r} \frac{\mathrm{I}}{2 a} \lambda
$$

Thus the typical solution for $r \varphi$ is

$$
r \varphi=\varepsilon^{-m r} \varepsilon^{n i(t-r \mid a)}
$$

in which $a=\sqrt{k \gamma}=$ the velocity of sound and the lower sign in (I7) has been chosen as corresponding to the outward traveling wave of diminishing intensity. An abitrary multiplier may be introduced and an arbitrary constant added to $t$. Omitting these and

${ }^{1}$ The absolute conductivity is taken as .00005572 as obtained by E. Müller, Wied. Ann., $60, \mathrm{II}_{3}$ (1896).

The other two roots of (16) ane very great and greater the smaller $v$; honce they do not correspond to the phy\$ical conditions of tise present problem. 
realizing the solution by discarding the imaginary part of the above expression we get for $V$

$$
\begin{gathered}
V=\frac{\partial}{\partial r}\left[\frac{\varepsilon^{-m r}}{r} \cos n\left(t-\frac{r}{a}\right)\right] \\
=\varepsilon^{-m r}\left[\frac{n}{\alpha r} \sin n\left(t-\frac{r}{a}\right)-\frac{\mathrm{I}+m r}{r^{2}} \cos n\left(t-\frac{r}{a}\right)\right]
\end{gathered}
$$

Thus the motion at any point consists in the superposition of two simple harmonic vibrations differing in phase by a quarter of a period. The ratio of the amplitude of the second to that of the first is

$$
m+\frac{\mathrm{I}}{r}: \frac{n}{a}
$$

Now $\frac{n}{a}$ is the modulus of the first approximation to $q$ and $m$ is the difference between the first and second approximations ${ }^{X}$; also $I / r$ rapidly diminishes as $r$ increases. Hence, after a short distance the motion is sensibly

$$
V=\frac{\varepsilon^{-m r}}{r} \sin n\left(t-\frac{r}{a}\right)
$$

Hence the intensity varies as

$$
\frac{\varepsilon^{-2 m r}}{r^{2}} \text {. }
$$

It will be seen from ( 18 ) that $m$ consists of three parts due to viscosity, conduction, and radiation respectively and that, while the first two increase very rapidly with increase of the vibration frequency, the third, due to radiation, is independent of the vibration frequency.

\section{Experimental.}

It is well known that great difficulties are encountered in any attempt to measure the intensity of sound or study its variations. In the following method the rate of decay of intensity is deduced from the distances at which sounds of different intrinsic intensity become inaudible and it is assumed that when two faint sounds are indis-

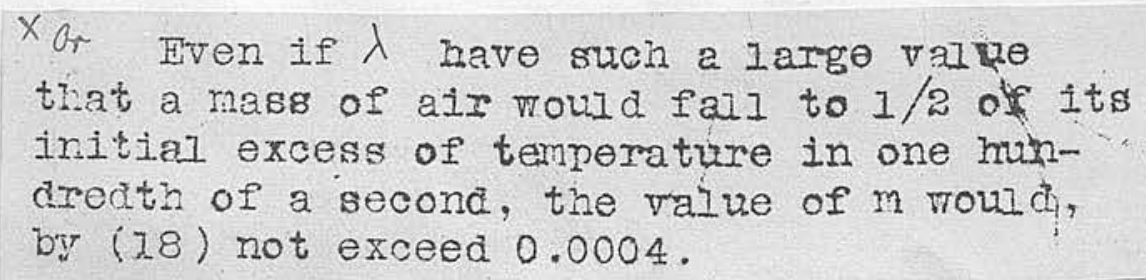


tinguishable by the ear as regards pitch and quality the minimum intensity required for audibility is the same for both.

A large number of very small whistles were made of as nearly as possible the same shape and dimensions. From these the eight that seemed most similar in all respects were chosen and mounted on a wind-chest in such a way that any number could be blown under a definite pressure indicated by a water manometer. The distances at which each pair and all the eight just became inaudible were then determined under conditions to be described presently. Let $R$ be the distance at which all eight whistles blown at once became inaudible and $r$ the mean distance at which two became inaudible. Then at a distance $r$ the mean intensity of two whistles equalled the minimum for audibility and hence that of the eight whistles equalled four times ${ }^{1}$ that minimum, while at distance $R$ the intensity of the eight whistles just equalled the minimum for audibility. Now it was found that near the limits of audibility the sounds were indistinguishable in quality. Hence, assuming (as stated above) that the minimum was the same in both cases, we have by the preceding theory

and

$$
\begin{array}{r}
\frac{\varepsilon^{-2 m R}}{\varepsilon^{-2 m r}} \cdot \frac{r^{2}}{R^{2}}=\frac{\mathrm{I}}{4} \\
m=\frac{\log _{e}\left(2 \frac{r}{R}\right)}{R-r}
\end{array}
$$

The observations were made at a very quiet place on the River St. John in New Brunswick, Canada, the whistles being sounded on

${ }^{1}$ It is here tacitly assumed that the intensity of the eight whistles equals the sum of their intensities when sounded separately. This may (if justification be needed) be justified on two grounds: Firstly, it was ascertained that the distance apart of the whistles on the wind-chest had no effect on the distance of audibility and that the quantity of energy spent in sounding all eight whistles (as measured by the wind expenditure at constant pressure) was the sum of the quantities expended in sounding them separately. Hence, the total energy of vibration produced is the sum of the quantities which would be produced by separate blowing. Secondly, that the intensities at any distance are also additive may be justified from the analysis. For in the case of the coexistence of several notes the solution for $V$ will be the sum of such partial solutions as (20), and by integrating $V \Delta p$ through a large multiple of the mean period it can be shown that the complex sound follows the same law of attenuation as the simple sounds ( $m$ being the mean of the values for the separate sources) and that the resultant mean intensity is the sum of the separate intensities. 
[YoL. VI.

one side of the river and the sounds listened to on the other side. Only times when there was no appreciable wind were considered suitable for work. To eliminate the effect of reflection from the banks several different stations for sounding and directions for observing were tried in different sets of observations. The orifices of the whistles were always kept turned directly toward the observer. To avoid errors due to tiring of the ear in some cases the observations on the pairs of whistles were made first and in other cases those on the eight whistles. Various devices were tried to eliminate the effect of bias in determining the distance of inaudibility. The difficulty of determining the point at which the sound became inaudible was found much less than was expected. Nevertheless, there was always a space in which it was doubtful whether the sound was heard or imagined. The middle of such a space was taken as the most probable position of extinction.

The relative intensities of the different pairs of whistles may be judged from the fact that in one case the distances of inaudibility were $508,5 \mathrm{II}, 5 \mathrm{I} 8,529$ meters and in another $622,629,636,647$ meters. These were the only cases in which the distance for each separate pair was finally measured. In general, the position for each pair was marked by a stake and the mean distance finally taken for measurement. In the absence of facilities for the purpose the pitch of the whistles was not determined until some weeks after the experiments were made. It was then found by using a high pressure sensitive flame to locate the nodes of the stationary waves produced by reflection from a wall. The semi-wave-lengths thus found at I $8^{\circ} \mathrm{C}$. were $2.49,2.46,2.4 \mathrm{I}, 2.4 \mathrm{I}, 2.46,2.42,2.42,2.44 \mathrm{cms}$. The mean of these is 2.44 , corresponding to a vibration frequency of 7000 .

In the following table of results the C. G. S. system of units is employed :

$\begin{array}{ccccc} & r & R & t & m \\ 1 & 38400 & 50600 & 27^{\circ} \mathrm{C} . & .000034 \\ 2 & 40700 & 51800 & 23 & .000040 \\ 3 & 63400 & 75400 & 15 & .000043 \\ 4 & 45800 & 55900 & 26 & .000049 \\ 5 & 59700 & 71200 & 15 & .000045 \\ & & & & \end{array}$


It may be noted that No. I was a preliminary experiment made with a view to finding the best conditions of work and was completed although a slight wind had sprung up which interfered with the observations. This probably accounts for the exceptional value of $m$. The very great differences in the values of $r$ and of $R$ on different occasions were due chiefly to wide variations in disturbing sounds such as those produced by birds and insects, the rustling of grass and the ripple of water.

It will be seen from this table that the eight whistles were, on an average, audible about one-fourth farther than a pair of whistles, whereas, if the sphericity of the waves had been the only cause of attenuation, the distance would have been as two to one. It will also be seen from the value of $2 m$ that the total energy of vibration decreases at about five-sixths of one per cent. per meter.

From (I 8) it can readily be calculated that for the high pitched notes employed the part of $m$ due to viscosity is .0000050 , and the part due to conduction .00000I9. The difference between the sum of these and the value for $m$ found experimentally is .000035. It will thus be seen what a relatively small part of the whole effect is due to viscosity and conduction, and what a large part is due to radiation and whatever other causes have not been considered. Of such hitherto unconsidered causes the only two that readily suggest themselves are atmospheric refraction (which causes a curvature of the lines of radiation) and reflections arising from a lack of homogeneity of the medium.

It does not, however, seem probable that either of these causes has contributed largely to the value of $m$. For, while viscosity, conduction, and radiation are comparatively constant in their action, refraction and internal reflection are causes which must vary very widely with circumstances of position and of the condition of the atmosphere and, had they been accountable for any considerable part of the effect observed, the separate values of $m$ could not have shown such a fair degree of accordance as they do. To make this clearer in regard to refraction, it may be noted that (I) there was practically no wind (the chief source of refraction), (2) the vertical temperature gradient (the only other considerable source of refraction) must have varied widely, since the time of observation varied 
from the heat of noonday to the cool of evening after sunset (in the last observation), (3) the height of the station at which the whistles were blown varied from three feet to nine feet above the level of the water, while the height of the observer's ear varied from six feet to ten feet.

Again, whatever the circumstances are that determine that lack of uniformity or "flocculence" of the atmosphere which was supposed by Tyndall to produce internal reflection, it seems highly probable that they varied widely because of (I) the variation of time of observation and of temperature already noted and (2) variations in the hygrometric state of the air ranging from comparative dryness to (in one case) saturation before a heavy shower, and as the values of $m$ show no such wide variations it seems improbable that much of the diminution of intensity was due to this cause. It would seem highly probable that, in any case, the air above a large body of water on a still day is comparatively uniform. No continuance of the sound after blowing had ceased could be observed, which is contrary to what would be expected if there had been much internal reflection. It may be noted also that internal reflections do not themselves produce a diminution of intensity in the case of a sustained note, for the reflection does not involve a diminution of energy of vibration, and reflections toward the observer will compensate for reflections away from him. Nevertheless the prolonging of the time of transmission from the source to the observer may lead to an increase in the effects due to viscosity, conduction, and radiation.

Assuming then that refraction and reflection contributed but little to the diminution of intensity observed, we see that radiation is about seven times as effective as viscosity and about eighteen times as effective as conduction in reducing the intensity of a note whose vibration frequency is 7000 . But the effects of viscosity and conduction are proportional to the square of the frequency, while that of radiation is the same for all sounds. Hence for a note of medium pitch, for example one whose vibration frequency is 350 , radiation must far transcend viscosity and conduction in effect, being in fact nearly 3000 times as effective as viscosity and over 7000 times as effective as conduction. The whole rate of diminution would, so far as these three causes are concerned, be practically that of radiation alone or about two-thirds of one per cent. per meter. 
It seems, therefore, improbable that, as some have thought, the mellowing of harsh sounds with distance is due to a higher rate of decay of the higher components. It is more probably due to their smaller initial energy which causes them, even with nearly the same rate of decay, to drop sooner to the limits of audibility.

\section{The Constant of Radiation of Air.}

According to Newton's law, the rate of cooling of a gas by radiation bears a constant ratio to the excess of its temperature above that of its surroundings. From the preceding experimental estimate of the effect of radiation on sound intensity and the theoretical value as given by equation (I8), it can be calculated that the value of this constant is for air about 8.3. It follows that a mass of air at any given excess of temperature above its surroundings will, if its volume remains constant, fall by radiation to one-half of that excess in about one-twelfth of a second. For reasons that appear in the preceding section this estimate of the rate of radiation must be regarded as more probably too great than too small.

Large as this estimate of the constant of radiation may seem to be, it is still not inconsistent with the known fact that the velocity of sound is (within the limits of experimental error) independent of the pitch. This can be seen by integrating (15) on the assumption that $\lambda$ is not negligible compared with $n$ or by calculation from the results of Stokes' discussion of the effect of radiation. Even for a note of as low frequency as 50 , it would only produce a decrease of velocity of one part in three thousand.

There is another respect in which the somewhat large value obtained for the constant of radiation is of interest. It would seem to point to an intimate association between gaseous particles and the ether, permitting of a rapid transfer of energy of vibration from the former to the latter.

Purdue University, Lafayette, Ind. 


\title{
PHYSICAL REVIEW \\ A JOURNAL OF EXPERIMENTAL AND THEORETICAL PHYSICS
}

\author{
CONDUCTED BY \\ EDWARD L. NICHOLS \\ ERNEST MERRITT, and FREDERICK BEDELL \\ XXXII
}

MARCH 1898

TABLE OF CONTENTS

The Attenuation of Sound and the Constant of Radiation of Air

A. WILIMER DUFF

Minor Contributions: ( $I$ ) On the Polarization of the Light Rellected from Hard Rubber. Alice H. Bruere. (2) On Latitude Variation in a Rigid Earth, as illustrated by Maxwell's Dynamical Top. Henry Crew. (3) Apparatus for Illustrating Potential Gradient. $R$. W. Wood. (4) Apparatus for Showing the Conductivity of Gases. $R$. W. Wood. (5) A Vacuum Tube to Illustrate the Slow Diffusion of the Residual Gases in High Vacua. Ernest Merritt.

New Books: Duhem: Méchanique Chimique. Anthony and Rrackett: Text-Book on Physics. Perry : Calculus for Engineers. Gerard: Electricity and Magnetism. Transactions of the American Institute of Electrical Engineers. Burnside. Theory of Groups. Engelmann: Tafeln und Tabellen zur Darstellung Spektroskopischer Beobachtungen. Schuster. Practical Physics. Austin and Thrwing: Physical Measurement. Wilberforce: Practical Physics Notebook. Wiedemann and Ebert: Physikalisches Praktikum. Merrill and Baldwin: Physics Course. Harrington: Physics for Grammar Schools. Hammel: Observation Blanks. Gage: Physical Experiments. Hall and Bergen: Text Book of Physics. Perry: Applied Mechanics. Christiansen: Theoretical Physics.

Copyright, 1898 , By The Macmillan Company

\author{
PUBLISHED FOR CORNELL UNIVERSITY \\ NEW YORK: THE MACMILLAN COMPANY \\ LONDON: MACMILLAN AND CO., LTD. \\ BERLIN MAYER AND MUELLER
}

